\title{
Correction to: Sustainability in the management of scientific information
}

\author{
Miguel Ángel Medina ${ }^{1,2,3}$ (D)
}

Published online: 6 November 2020

(C) Springer Japan KK, part of Springer Nature 2020

\section{Correction to: Sustainability Science https://doi.org/10.1007/s11625-020-00864-0}

In the original publication of the article, under the introduction section the following sentence "This avalanche of data is causing the growing gap..." was published incorrectly. The correct sentence should read as "This avalanche of data is causing a growing gap...".

In addition, under the section The Red Queen effect in scientific activity, the following sentence "In 1988 we publish an article in one of the most prestigious journals..." was incorrect. The correct sentence should read as "In 1988 we published an article in one of the most prestigious journals...".

Further, under the section Good science needs time to mature, the following sentence "..., in the race to publish the more, the higher and the sooner, few..." was incorrect. The correct sentence should read as "..., in the race to publish the most, the highest and the soonest, few...".

Under the section, Who decides what science deserves to be published?, the following sentence "As the Spanish say goes: We were a few and Grandma gave birth!..." should read as "As the Spanish saying goes: We were a few and Grandma gave birth!".

The original article can be found online at https://doi.org/10.1007/ s11625-020-00864-0.

Miguel Ángel Medina

medina@uma.es

1 Departamento de Biología Molecular y Bioquímica, Facultad de Ciencias, Universidad de Málaga, Andalucía Tech, 29071 Málaga, Spain

2 IBIMA (Biomedical Research Institute of Málaga), 29071 Málaga, Spain

3 CIBER de Enfermedades Raras (CIBERER), 29071 Málaga, Spain
Finally, under the Box 2: The Six Principles of Slow Science, we found some typographical errors and corrections are provided below.

- In 4th point of Box 2, the following sentence "Scientific knowledge is at the disposal of all humans on the planter." Should read as "Scientific knowledge is at the disposal of all humans on the planet."

- In 4(b) of Box 2, the following sentence "... commodify and monopolize scientific knwledge, ..." should read as “... commodify and monopolize scientific knowledge, ..."

- In 4(c) of Box 2, the following sentence "... and development -science divelops ..." should read as "... and development -science develops ..."

- In 5(d) of Box 2, the following sentence "... long-term fundamental and relevan topics ..." should read as “... long-term fundamental and relevant topics ..."

- In 6th point of Box 2, the following sentence " ... even in a competitiveglobal market ..." should read as "... even in a competitive global market ..."

The original version was updated. 